\title{
Complexity of quantum motion and quantum-classical correspondence: A phase-space approach
}

\author{
Jiaozi Wang $\odot,{ }^{1, *}$ Giuliano Benenti $\odot, 2,3,4, \dagger$ Giulio Casati, ${ }^{2,5, \$}$ and Wen-ge Wang $\oplus^{1, \S}$ \\ ${ }^{1}$ Department of Modern Physics, University of Science and Technology of China, Hefei 230026, China \\ ${ }^{2}$ Center for Nonlinear and Complex Systems, Dipartimento di Scienza e Alta Tecnologia, Università degli Studi dell'Insubria, \\ via Valleggio 11, 22100 Como, Italy \\ ${ }^{3}$ Istituto Nazionale di Fisica Nucleare, Sezione di Milano, via Celoria 16, 20133 Milano, Italy \\ ${ }^{4}$ NEST, Istituto Nanoscienze-CNR, I-56126 Pisa, Italy \\ ${ }^{5}$ International Institute of Physics, Federal University of Rio Grande do Norte, Campus Universitário - Lagoa Nova, \\ CP. 1613, Natal, Rio Grande Do Norte 59078-970, Brazil
}

(Received 5 January 2020; accepted 12 October 2020; published 3 November 2020)

\begin{abstract}
We discuss the connection between the out-of-time-ordered correlator and the number of harmonics of the phase-space Wigner distribution function. In particular, we show that both quantities grow exponentially for chaotic dynamics, with a rate determined by the largest Lyapunov exponent of the underlying classical dynamics, and algebraically_-linearly or quadratically — for integrable dynamics. It is then possible to use such quantities to detect in the time domain the integrability-to-chaos crossover in many-body quantum systems.
\end{abstract}

DOI: 10.1103/PhysRevResearch.2.043178

\section{INTRODUCTION}

Understanding, characterizing, and measuring the complexity of (many-body) quantum dynamics is a fundamental problem, also of great practical relevance for the prospects of quantum information science [1] and more generally of quantum technologies. In particular, the out-of-time-ordered correlator (OTOC) (see, e.g., [2-30]) has been put forward as a measure of chaos in many-body quantum systems [3], and it has been related to mixing (called scrambling when referring to quantum information) and thermalization, in different contexts, ranging from condensed matter [2] to black hole physics [3-5].

In classical mechanics a well-defined notion of complexity exists, based on local exponential instability of trajectories, that is, on the positivity of the largest Lyapunov exponent. In turn, this implies positive algorithmic complexity [31,32], so that orbits are in practice unpredictable, and the memory of initial conditions is lost [33].

The above notion of complexity cannot be readily transferred to quantum mechanics, where trajectories cannot be defined due to the Heisenberg uncertainty principle. On the other hand, complexity can be treated on equal footing for classical and quantum mechanics in phase space, see [34-45] for first attempts in this direction. The exponential sensitivity

\footnotetext{
*wangjz@mail.ustc.edu.cn

† giuliano.benenti@uninsubria.it

‡iulio.casati@uninsubria.it

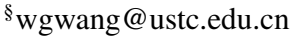

Published by the American Physical Society under the terms of the Creative Commons Attribution 4.0 International license. Further distribution of this work must maintain attribution to the author(s) and the published article's title, journal citation, and DOI. to initial conditions implies that the density distribution in the phase space is exponentially stretched and folded, and therefore becomes complex and intricate on ever smaller and smaller scales. Therefore, if one wants to reconstruct numerically the increasingly finer details of the phase-space distribution, it is intuitive to expect that the number of harmonics (i.e., components in Fourier space) that are excited also increases exponentially in time $[35,38,39]$. In an integrable system this does not happen, as the instability is typically linear in time [46]. It follows that the growth rate of the number of harmonics of the phase-space distribution can be used, similarly to the Lyapunov exponent, as a way to characterize classical chaos.

One might object that there is the problem of choosing the phase-space coordinates. However, while the finite-time results of course depend on the basis, the asymptotic growth rate does not, as it is given by the largest Lyapunov exponent [47], which is base-independent. If we consider action-angle variables of an unperturbed, integrable system, then a nonintegrable perturbation will lead to an exponential growth of the number of harmonics with a rate given by the largest Lyapunov exponent. On the other hand, an integrable perturbation may distort even strongly unperturbed tori, so that at short times a large number of harmonics may be excited, but asymptotically the number of harmonics will grow linearly in time.

The phase-space approach can be conveniently generalized to quantum mechanics, using the number of harmonics of the Wigner function as a suitable measure of the complexity of a quantum state.

In this paper we study the number of harmonics and the OTOC, both for classically chaotic and integrable systems. We show that both quantities can be used to detect, in the time domain, the crossover from integrability to chaos. Moreover, we show that the number of harmonics and the OTOC are connected. Such connection places the OTOC on a broader 
context and reinforces its interpretation as a complexity quantifier. The above results are illustrated numerically for the case of two nonlinearly coupled oscillators.

\section{THEORETICAL BASIS}

We start by considering the number of harmonics in classical mechanics. Let $H=H_{0}+H_{I}$ be the Hamiltonian of a $N$-particle system, where $H_{0}$ is time-independent and integrable, while $H$ might be either integrable or nonintegrable. We write the classical distribution function $\rho(\boldsymbol{I}, \boldsymbol{\theta} ; t)$ in terms of the action-angle variables of $H_{0}, \boldsymbol{I}=\left(I_{1}, \ldots, I_{N}\right)$ and $\boldsymbol{\theta}=$ $\left(\theta_{1}, \ldots, \theta_{N}\right)$. Taking the Fourier transform

$$
\rho(\boldsymbol{I}, \boldsymbol{\theta} ; t)=\frac{1}{\pi^{N}} \sum_{\boldsymbol{m}} \rho_{\boldsymbol{m}}(\boldsymbol{I} ; t) \exp (i \boldsymbol{m} \cdot \boldsymbol{\theta}),
$$

we define the second moment of the harmonics distribution as

$$
\mathcal{M}_{2}^{c l}(t)=\frac{\sum_{\boldsymbol{m}} \boldsymbol{m}^{2} \int_{0}^{\infty} d I\left|\rho_{\boldsymbol{m}}(\boldsymbol{I} ; t)\right|^{2}}{\sum_{\boldsymbol{m}} \int_{0}^{\infty} d I\left|\rho_{\boldsymbol{m}}(\boldsymbol{I} ; t)\right|^{2}} .
$$

Making use of the properties of the Fourier transform, $\mathcal{M}_{2}^{c l}(t)$ can also be written as

$$
\mathcal{M}_{2}^{c l}(t)=\frac{\int d \boldsymbol{I} d \boldsymbol{\theta} \sum_{k}\left|\frac{\partial}{\partial \theta_{k}} \rho(\boldsymbol{I}, \boldsymbol{\theta} ; t)\right|^{2}}{\int d \boldsymbol{I} d \boldsymbol{\theta}|\rho(\boldsymbol{I}, \boldsymbol{\theta} ; t)|^{2}} .
$$

Due to the Hamiltonian evolution, the denominator of Eq. (3) is constant in time. So the behavior of $\mathcal{M}_{2}^{c l}(t)$ is determined by the numerator, notably by the behavior of $\frac{\partial}{\partial \theta_{k}} \rho(\boldsymbol{I}, \boldsymbol{\theta} ; t)$ for different $k$. We obtain [48]

$$
\mathcal{M}_{2}^{c l}(t)=\frac{\int d \chi(0)\left|\widetilde{\nabla} \rho_{0}[\chi(0)]\right|^{2} \sum_{k=1}^{N}\left[\delta I_{k}(t)\right]^{2}}{d^{2} \int d \chi(0)\left|\rho_{0}[\chi(0)]\right|^{2}},
$$

where $\chi(t)=\left[\theta_{1}(t), \ldots, \theta_{N}(t), I_{1}(t), \ldots, I_{N}(t)\right]$ is the actionangle vector at time $t, \widetilde{\nabla}=\left(\frac{\partial}{\partial I_{1}}, \ldots, \frac{\partial}{\partial I_{N}},-\frac{\partial}{\partial \theta_{1}}, \ldots,-\frac{\partial}{\partial \theta_{N}}\right)$ a modified gradient, $\rho_{t}[\chi(t)]$ the classical distribution function, and $\delta I_{k}(t)$ indicates the deviation of the $k$ th action at time $t$, for two points in phase space, whose initial positions are $\chi(0)$ and $\chi(0)+\delta \chi(0)$, respectively, with $\delta \chi(0)=d \frac{\tilde{\nabla} \rho_{0}[\chi(0)]}{\left|\tilde{\nabla} \rho_{0}[\chi(0)]\right|}$ and $d \rightarrow 0$. From Eq. (4) one finds that the behavior of the number of harmonics in classical case is determined by the quantities $\delta I_{k}(t)$. In the integrable case, $\delta I_{k}(t)$ typically increases linearly with time, and thus $\mathcal{M}_{2}^{c l}(t)$ has a quadratic growth. For the chaotic case, $\delta I_{k}(t)$ increases exponentially with time, and so is the case for $\mathcal{M}_{2}^{c l}(t)$. For a numerical confirming evidence, see below.

In the quantum case, for the sake of simplicity we limit ourselves to systems whose Hamiltonian $\hat{H}$ can be written in terms of a set of bosonic creationannihilation operators. That is, $\hat{H}=\hat{H}_{0}+\hat{H}_{1}$, where $\hat{H}_{0}=\hat{H}_{0}\left(\hat{n}_{1}, \ldots, \hat{n}_{N}\right)$ is time-independent and integrable, while $\hat{H}_{1}=\hat{H}_{1}\left(\hat{a}_{1}^{\dagger}, \ldots, \hat{a}_{N}^{\dagger}, \hat{a}_{1}, \ldots, \hat{a}_{N} ; t\right)$, with $\left[\hat{a}_{i}, \hat{a}_{j}\right]=$ $\left[\hat{a}_{i}^{\dagger}, \hat{a}_{j}^{\dagger}\right]=0, \quad\left[\hat{a}_{i}^{\dagger}, \hat{a}_{j}\right]=\delta_{i j}$, and the number operators $\hat{n}_{i}=\hat{a}_{i}^{\dagger} \hat{a}_{i}$. The Wigner function of a state, which is described by a density operator $\hat{\rho}(t)$, can be written as [49-51]

$$
\begin{aligned}
W\left(\boldsymbol{\alpha}, \boldsymbol{\alpha}^{*} ; t\right)= & \frac{1}{\pi^{2 N} \hbar^{N}} \int d^{2} \boldsymbol{\eta} \exp \left(\frac{\boldsymbol{\eta}^{*} \cdot \boldsymbol{\alpha}}{\sqrt{\hbar}}-\frac{\boldsymbol{\eta} \cdot \boldsymbol{\alpha}^{*}}{\sqrt{\hbar}}\right) \\
& \times \operatorname{Tr}[\hat{\rho}(t) \hat{D}(\boldsymbol{\eta})],
\end{aligned}
$$

where $\boldsymbol{\eta}=\left(\eta_{1}, \ldots, \eta_{N}\right)$ and $\boldsymbol{\alpha}=\left(\alpha_{1}, \ldots, \alpha_{N}\right)$ are $N$ dimensional complex variables, and the displacement operator

$$
\hat{D}(\boldsymbol{\eta})=\exp \left[\sum_{i=1}^{N}\left(\eta_{i} \hat{a}_{i}^{\dagger}-\eta_{i}^{*} \hat{a}_{i}\right)\right]
$$

We then consider the Fourier expansion of the Wigner function,

$$
W\left(\boldsymbol{\alpha}, \boldsymbol{\alpha}^{*} ; t\right)=\frac{1}{\pi^{N}} \sum_{\boldsymbol{m}} W_{\boldsymbol{m}}(\boldsymbol{I} ; t) \exp (i \boldsymbol{m} \cdot \boldsymbol{\theta}),
$$

where $I_{k}$ and $\theta_{k}$ are related to $\alpha_{k}$ by $\alpha_{k}=\frac{q_{k}+i p_{k}}{\sqrt{2}}=\sqrt{I_{k}} e^{i \theta_{k}}$. Here $I_{k}$ and $\theta_{k}$ can be regarded as a polar coordinates in the $\left(q_{k}, p_{k}\right)$ plane. The number of harmonics is estimated by $\sqrt{\mathcal{M}_{2}}$, where $\mathcal{M}_{2}(t)=\sum_{\boldsymbol{m}}|\boldsymbol{m}|^{2} \mathcal{W}_{\boldsymbol{m}}(t)$ is the second moment of the harmonics distribution, with

$$
\mathcal{W}_{\boldsymbol{m}}(t)=\frac{\int d \boldsymbol{I}\left|W_{\boldsymbol{m}}(\boldsymbol{I} ; t)\right|^{2}}{\sum_{\boldsymbol{m}} \int d \boldsymbol{I}\left|W_{\boldsymbol{m}}(\boldsymbol{I} ; t)\right|^{2}} .
$$

In terms of density matrix $\hat{\rho}(t)$, we can also write $[35,38,40,42]$

$$
\mathcal{W}_{\boldsymbol{m}}(t)=\frac{\sum_{n_{k}>0}|\langle\boldsymbol{n}+\boldsymbol{m}|\hat{\rho}(t)| \boldsymbol{n}\rangle|^{2}}{\sum_{n_{k}>0,} \sum_{\left(m_{k}+n_{k}\right)>0}|\langle\boldsymbol{n}+\boldsymbol{m}|\hat{\rho}(t)| \boldsymbol{n}\rangle|^{2}} .
$$

Following [35,39], $\mathcal{M}_{2}(t)$ can be written as

$$
\mathcal{M}_{2}(t)=\frac{\sum_{k} \operatorname{Tr}\left(\left|\left[\hat{\rho}(t), \hat{I}_{k}\right]\right|^{2}\right)}{\hbar^{2} \operatorname{Tr}\left[\hat{\rho}^{2}(t)\right]},
$$

where $\hat{I}_{k}=\hbar \hat{n}_{k}$. In the case of a pure state $|\psi(t)\rangle$,

$$
\mathcal{M}_{2}(t)=\frac{2 \sum_{k}\left[\Delta I_{k}(t)\right]^{2}}{\hbar^{2}},
$$

where

$$
\left[\Delta I_{k}(t)\right]^{2}=\left\langle\psi(t)\left|\hat{I}_{k}^{2}\right| \psi(t)\right\rangle-\left\langle\psi(t)\left|\hat{I}_{k}\right| \psi(t)\right\rangle^{2}
$$

is the variance for measurement at time $t$, corresponding to observable $\hat{I}_{k}$.

We now turn to OTOC, defined as the expectation of the square commutator of two operators taken at different times:

$$
\mathcal{C}(t)=\left\langle|[\hat{A}(t), \hat{B}(0)]|^{2}\right\rangle .
$$

It is interesting to remark that the numerator of the right-hand side (rhs) of Eq. (10), which measures the number of harmonics (the denominator is constant in time for Hamiltonian evolution), turns out to be a particular kind of OTOC, with $\hat{A}(t)=\hat{\rho}(t)$ and $\hat{B}(0)=\hat{I}_{k}$, and the average taken over the whole Hilbert space. In the following, however, we consider a more commonly used expression for OTOC,

$$
C_{p p}(t)=-\frac{1}{\hbar^{2}}\left\langle\psi_{0}\left|\left[\hat{p}_{1}(t), \hat{p}_{1}(0)\right]^{2}\right| \psi_{0}\right\rangle,
$$

where $\hat{p}_{1}$ is the momentum operator for one particle (denoted as particle 1) and $\left|\psi_{0}\right\rangle$ is the initial state (at time $t=0$ ) of the composite, $N$-particle system.

The classical correspondence of $C_{p p}(t)$, denoted as $C_{p p}^{c l}(t)$, is obtained by the canonical substitution $\frac{1}{i \hbar}[\hat{A}, \hat{B}] \rightarrow\{A, B\}_{\mathrm{PB}}$, 
where the right-hand side is the Poisson bracket of the classical variables $A$ and $B$. We obtain

$$
\begin{aligned}
C_{p p}^{c l}(t) & =\int d \boldsymbol{\gamma}(0) \rho_{0}[\boldsymbol{\gamma}(0)]\left\{p_{1}(t), p_{1}(0)\right\}_{P B}^{2} \\
& =\int d \boldsymbol{\gamma}(0) \rho_{0}[\boldsymbol{\gamma}(0)]\left(\frac{\delta p_{1}(t)}{\delta q_{1}(0)}\right)^{2},
\end{aligned}
$$

where $\quad \boldsymbol{\gamma}(t)=\left[q_{1}(t), \ldots, q_{N}(t), p_{1}(t), \ldots, p_{N}(t)\right] \quad$ and $\rho_{0}[\boldsymbol{\gamma}(0)]$ is the initial distribution of the classical ensemble corresponding, in the quantum case, to the Wigner function of the initial state. It is clear from Eq. (15) that the behavior of $C_{p p}^{c l}(t)$ is determined by the quantity $\left(\frac{\delta p_{1}(t)}{\delta q_{1}(0)}\right)^{2}$. In the integrable case, $\delta p_{1}(t)$ increases linearly with time, and therefore $C_{p p}^{c l}(t)$ is expected to be a quadratic function of time, while in the chaotic case $\delta p_{1}(t)$ has an exponential growth, and so is $C_{p p}^{c l}(t)$.

Barring subtle points related to the phase-space averaging procedure [48], we can conclude that both $\mathcal{M}_{2}^{c l}(t)$ and the OTOC $C_{p p}^{c l}(t)$ grow exponentially for chaotic classical systems, with a rate given by twice the largest Lyapunov exponent $\lambda_{L}\left(\boldsymbol{r}_{0}\right)$ of the trajectory started at the center of the initial distribution, assumed to be narrow:

$$
\mathcal{M}_{2}^{c l}(t), C_{p p}^{c l}(t) \propto \exp \left[2 \lambda_{L}\left(\boldsymbol{r}_{0}\right) t\right] .
$$

While the short-time behavior depends on the chosen phasespace coordinates, the growth rate, which is determined by the largest Lyapunov exponent, does not.

\section{NUMERICAL RESULTS}

To illustrate the above analytical results, we consider a model of two coupled oscillators, whose Hamiltonian is

$$
\hat{H}=\frac{1}{2}\left(\hat{p}_{1}^{2}+\hat{q}_{1}^{2}\right)+\frac{1}{2}\left(\hat{p}_{2}^{2}+\hat{q}_{2}^{2}\right)+\frac{\beta}{4}\left(\hat{q}_{1}^{4}+\hat{q}_{2}^{4}\right)+\frac{1}{2} \hat{q}_{1}^{2} \hat{q}_{2}^{2},
$$

where $\hat{H}_{0}$ is written as

$$
\hat{H}_{0}=\frac{1}{2}\left(\hat{p}_{1}^{2}+\hat{q}_{1}^{2}\right)+\frac{1}{2}\left(\hat{p}_{2}^{2}+\hat{q}_{2}^{2}\right) .
$$

This model allows us to investigate both the integrable case $\beta=1$, the chaotic case $\beta \ll 1$, and the crossover between the two regimes. As initial state $\left|\psi_{0}\right\rangle$ we consider the tensor product of Gaussian wave packets for both particles. We average our numerical data over $\mathcal{N}$ initial conditions, with the centers of the (Gaussian) Wigner functions randomly distributed within the energy shell $\left[E_{0}-\delta E / 2, E_{0}+\delta E / 2\right]$, with $\delta E \ll E$. In our dimensionless units the width in $q_{k}$ and $p_{k}(k=1,2)$ of the initial Wigner functions is proportional to $\sqrt{\hbar}$, with $\hbar$ effective Planck constant. In the classical case, we average over the corresponding initial Gaussian distributions [52].

Numerical results for the number of harmonics and the OTOC are shown in Figs. 1 and 2, respectively. Here we average over different initial states as follows:

$$
\overline{\ln \mathcal{M}_{2}(t)}=\frac{1}{\mathcal{N}} \sum_{k=1}^{\mathcal{N}} \ln \mathcal{M}_{2}^{(k)}(t), \quad \overline{\ln C_{p p}(t)}=\frac{1}{\mathcal{N}} \sum_{k=1}^{\mathcal{N}} \ln C_{p p}^{(k)}(t),
$$
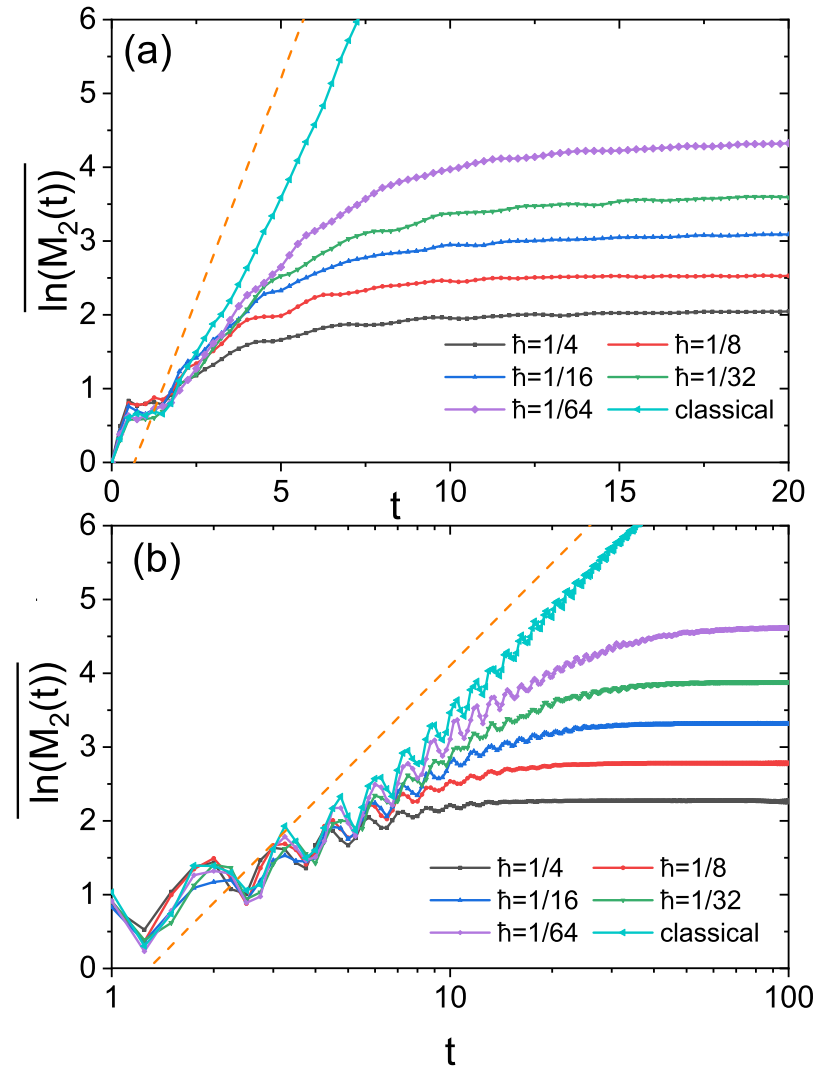

FIG. 1. Dependence of the averaged number of harmonics on time for different value of $\hbar$ and in the classical case (a) in the chaotic regime for $\beta=0$ (the dashed line indicates the exponential growth of $\mathcal{M}_{2}$, with rate 1.2 equal to two times a generalized largest Lyapunov exponent); (b) in the integrable regime $\beta=1$, where $t$ is given in logarithmic scale (the dashed line indicates the quadratic growth of $\mathcal{M}_{2}$ ). In the classical case we average over 100 different initial ensembles and in the quantum case over 100 initial states, distributed randomly within the energy shell with $E_{0}=8, \delta E=0.002$. To avoid effects due to the size of $\hbar$, which are not relevant to determine the growth rate, here we subtract the $t=0$ value.

where $\mathcal{M}_{2}^{(k)}(t)$ and $C_{p p}^{(k)}(t)$ are the number of harmonics and OTOC starting from the $k$ th initial condition, and $\mathcal{N}$, as discussed above, is the number of initial conditions we consider within the energy shell. Such averaging addresses the problem of spurious exponential growth in integrable systems, due to unstable fixed points in phase space rather than to chaos $[30,56]$. With the averaging (19), we can unambiguously associate the exponential (quadratic) growth of $\mathcal{M}_{2}(t)$ and $C_{p p}(t)$ to chaos (integrability). Indeed, in agreement with theoretical predictions, it is seen that both quantities, in the semiclassical regime and for chaotic dynamics, grow exponentially, with a rate determined by the largest Lyapunov exponent $\lambda_{L}[47,48]$. On the other hand, the growth is quadratic when the system is integrable. In all cases, it is clear that the quantum results converge to the classical ones when the effective Planck constant $\hbar \rightarrow 0$. For the OTOC, it appears that the quantum to classical correspondence, as expected, is valid up to the logarithmic time scale in the chaotic case $t_{E} \sim \ln (1 / \hbar) / \lambda_{L}$ [53], while in the integrable case the correspondence is valid up to a much longer time, growing as $1 / \hbar$. 

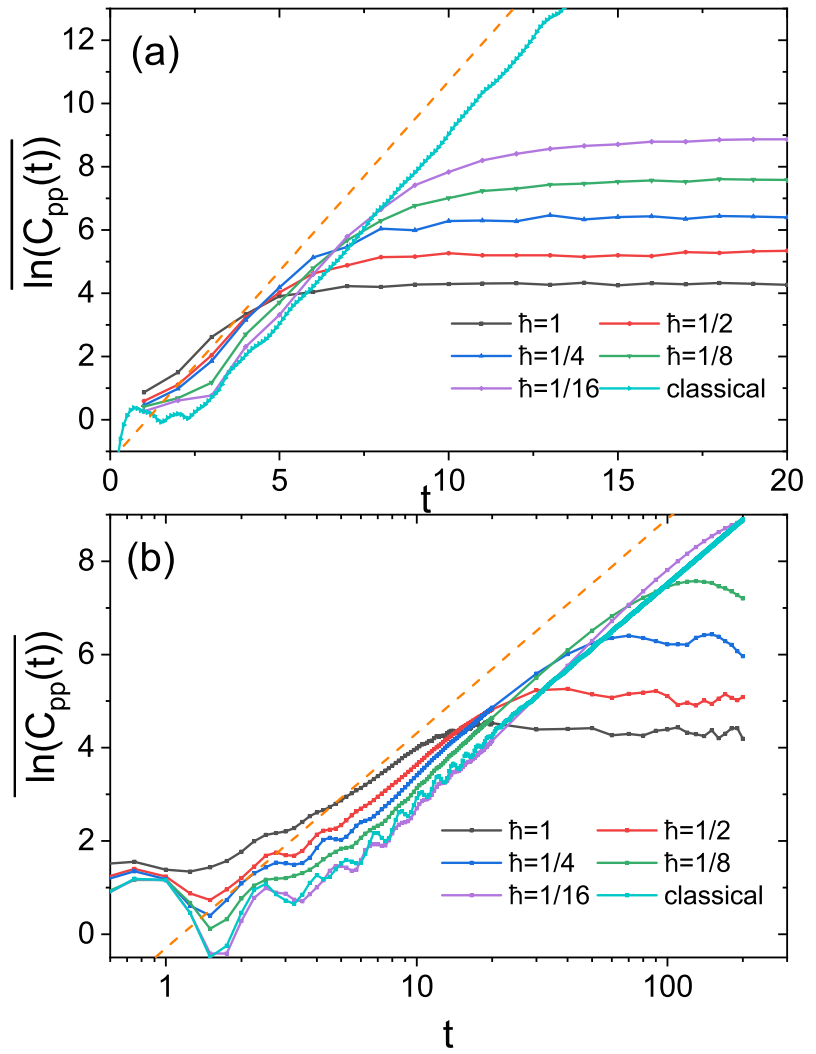

FIG. 2. Dependence of averaged OTOC on time for different value of $\hbar$ and in the classical case, (a) in the chaotic regime for $\beta=0$; (b) in the integrable regime $\beta=1$. In the classical case, the results are averaged over 100 different initial ensemble and in quantum case over 100 initial states, respectively, distributed randomly in the same energy shell as in the previous figure. The dashed lines indicate exponential (top) and quadratic (bottom) growth. In the chaotic case, the growth rate is the same as the one in Fig. 1 for the number of harmonics.

With regard to the number of harmonics, it can be seen that the convergence of quantum results to the classical ones requires smaller values of $\hbar$. The deviation of $\mathcal{M}_{2}(t)$ from $\mathcal{M}_{2}^{c l}(t)$ is determined by the deviation of the Wigner function from the corresponding classical distribution function. Though we consider initial coherent states, for which the Wigner function is everywhere positive and exactly equal to the corresponding classical distribution, nonlinear dynamics generates negative fringes in the Wigner function, which usually deviates from the classical distribution function at times much shorter than the Ehrenfest time. Thus to reveal the integrable or chaotic nature of dynamics from the quadratic or exponentially growth of the number of harmonics, one needs a smaller $\hbar$ than for the OTOC.

The different time dependence between integrable and chaotic regime for number of harmonics and OTOC suggests that these quantities can be used to detect the crossover from integrability to chaos in the time domain. To provide a clear evidence of this expectation, we plot in Fig. 3 an average velocity $\bar{v}_{o}=\overline{C_{p p}}\left(t_{o}^{\star}\right) / t_{o}^{\star}$, where $t_{o}^{\star}$ indicates the time at which $\overline{C_{p p}}$ reaches half of its saturation value. The figure shows that the crossover to chaos takes place in the region around

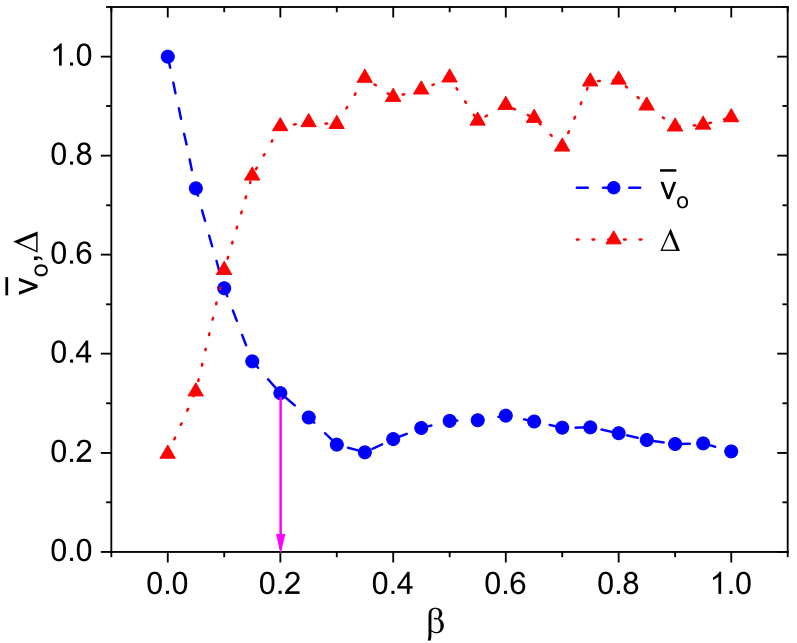

FIG. 3. Average velocity $\bar{v}_{o}$ in the growth of OTOC and distance to chaos measured by $\Delta$. Data for $\Delta$ are for $\hbar=1 / 64$, while for $\bar{v}_{o} \hbar=1 / 8$. To get a better comparison of the results, $\bar{v}_{o}$ is rescaled to its maximal value. The arrow roughly indicates the value of $\beta$ for the transition to chaos.

$\beta=0.2$. This is in agreement with results obtained from energy-level statistics [54], which is the ordinary tool to detect such a transition. In the later case, we consider a parameter $\Delta$, defined as

$$
\Delta=\frac{\int_{0}^{\infty}\left|P(s)-P_{w}(s)\right| d s}{\int_{0}^{\infty}\left|P_{p}(s)-P_{w}(s)\right| d s},
$$

where $P(s)$ is the nearest-level-spacing distribution of the system, and $P_{w}(s)\left[P_{p}(s)\right]$ is the Wigner-Dyson (Poisson) distribution. The quantity $\Delta$ measures the distance between the level spacings distribution of our model and the Poisson and Wigner-Dyson distributions, normalized in such a way that $\Delta=0$ corresponds to Wigner-Dyson and $\Delta=1$ to Poisson distribution. Variation of $\Delta$ versus $\beta$ shows the same crossover region around $\beta=0.2$.

\section{DISCUSSION AND CONCLUSION}

We showed that both the number of harmonics and OTOC are convenient tools to characterize complexity in classical and quantum mechanics. Both quantities can be used, similarly to spectral statistics, to detect the crossover from integrability to chaos. In contrast with spectral statistics, which can only provide information on the integrable or chaotic nature of dynamics, OTOC and number of harmonics also measure the "strength" of chaos, as provided by the largest Lyapunov exponent. In this connection, we recall that nonintegrable systems with zero Lyapounov exponent (e.g., irrational polygonal billiards) exhibit Wigner-Dyson statistics.

While in classical mechanics the (exponential or quadratic) growth of both quantities continues forever, in quantum mechanics it eventually saturates due to the discreteness of quantum phase space, i.e., its noncommutative geometry. In particular, for chaotic dynamics and in the semiclassical regime the saturation value of number of harmonics and OTOC can be obtained using the eigenstate thermalization hypothesis [55]. We obtain [48] $\overline{\ln \mathcal{M}_{2}(\infty)}, \overline{\ln C_{p p}(\infty)} \propto-\ln \hbar$. 
Note that, as the growth of number of harmonics and OTOC is exponential in time, saturation takes place on the timescale logarithmically short in $\hbar$.

It is also interesting to comment on the relationship between OTOC and number of harmonics. While in the quantum case the link can be understood by interpreting the second moment of the harmonics distribution as a particular kind of OTOC, it is the phase-space picture which allows a more intuitive connection. When considering canonical variables, the classical OTOC is the phase-space average of their squared Poisson bracket, which in turn is related to exponential sensitivity of trajectories, see Eq. (15) for the case of $C_{p p}$. On the other hand, the number of harmonics can be seen as the phase-space average of $\sum_{k}\left[\delta I_{k}(t)\right]^{2}$, with $I_{k}$ action variables for the unperturbed Hamiltonian, over a modified distribution function, see Eq. (4). For both quantities, it is the integrable or nonintegrable nature of the perturbation which determines their growth in time. While the relationship between OTOC and number of harmonics is generic, an intuitive understanding of their connection on the basis of the phase-space approach still has to be developed for many-body quantum systems without classical analog, i.e., for quantum spin chains.

The last point we want to mention here is that the second moment $\mathcal{M}_{2}(t)$ of the harmonics distribution can, in principle, be measured experimentally, by making use of the multiple quantum coherence (MQC) experiments, which is a method already used to measure OTOC [57]. To measure $\mathcal{M}_{2}(t)$, one can adapt the method of [58]. To simplify writing, we consider the one-dimensional case. Let $|n\rangle$ be the eigenstates of the number operator $\hat{n}: \hat{n}|n\rangle=n|n\rangle$. One can divide the density matrix of an arbitrary state $\hat{\rho}(t)$ into blocks as $\hat{\rho}(t)=$ $\sum_{m} \hat{\rho}_{m}(t)$, where

$$
\hat{\rho}_{m}(t)=\sum_{n-n^{\prime}=m} \rho_{n n^{\prime}}(t)|n\rangle\left\langle n^{\prime}\right| .
$$

The Frobenius norm $\quad I_{m}[\hat{\rho}(t)]=\left(\left\|\hat{\rho}_{m}(t)\right\|\right)^{2}=$ $\operatorname{tr}\left[\hat{\rho}_{m}^{\dagger}(t) \hat{\rho}_{m}(t)\right]=\sum_{n-n^{\prime}=m}\left|\rho_{n n^{\prime}}(t)\right|^{2}$, is related to $\mathcal{W}_{m}(t)$ [see Eq. (9)] as follows:

$$
I_{m}[\hat{\rho}(t)]=\frac{\mathcal{W}_{m}(t)}{\operatorname{tr}\left[\hat{\rho}^{2}(t)\right]} .
$$

To measure $I_{m}[\hat{\rho}(t)]$, one can evovle $\hat{\rho}_{0}$ into $\hat{\rho}(t)$, apply $\hat{W}(\phi)=e^{-i \hat{n} \phi}$, evolve backward (echo experiment) to $\hat{\rho}_{f}$, and measure the probability to find the system in the initial state, given by $\operatorname{tr}\left[\hat{\rho}_{0} \hat{\rho}_{f}\right]$. Since $\hat{W}(\phi) \hat{\rho}_{m}(t) \hat{W}^{\dagger}(\phi)=e^{i m \phi} \hat{\rho}_{m}(t)$, we obtain

$$
\operatorname{tr}\left[\hat{\rho}_{0} \hat{\rho}_{f}\right]=\sum_{m} I_{m}[\hat{\rho}(t)] e^{-i m \phi} .
$$

Finally, by Fourier transforming the signal with respect to $\phi$, one obtains the MQC spectrum $\left\{I_{m}[\hat{\rho}(t)]\right\}$, and the second moment of the harmonics distribution:

$$
\mathcal{M}_{2}(t)=\operatorname{tr}\left[\hat{\rho}_{m}^{2}(t)\right] \sum_{m} m^{2} I_{m}[\hat{\rho}(t)] .
$$

\section{ACKNOWLEDGMENTS}

J.W. and W.-g.W. acknowledge the Natural Science Foundation of China under Grants No. 11535011 and No. 11775210. G.B. acknowledges the financial support of the INFN through the project "QUANTUM."
[1] G. Benenti, G. Casati, D. Rossinii, and G. Strini, Principles of Quantum Computation and Information (A Comprehensive Textbook) (World Scientific, Singapore, 2019).

[2] A. I. Larkin and Yu. N. Ovchinnikov, Sov. Phys. JETP 28, 1200 (1969).

[3] A. Kitaev, Hidden Correlations in the Hawking Radiation and Thermal Noise, talk given at KITP, Santa Barbara, 2014, http: //online.kitp.ucsb.edu/online/joint98/kitaev/.

[4] J. Maldacena and D. Stanford, Phys. Rev. D 94, 106002 (2016).

[5] J. Maldacena, S. H. Shenker, and D. Stanford, J. High Energy Phys. 08 (2016) 106.

[6] P. Hosur, X.-L. Qi, D. A. Roberts, and B. Yoshida, J. High Energ. Phys. 02 (2016) 004.

[7] E. B. Rozenbaum, S. Ganeshan, and V. Galitski, Phys. Rev. Lett. 118, 086801 (2017).

[8] I. Kukuljan, S. Grozdanov, and T. Prosen, Phys. Rev. B 96, 060301(R) (2017)

[9] R. Fan, P. Zhang, H. Shen, and H. Zhai, Sci. Bull. 62, 707 (2017).

[10] M. Giärttner, J. G. Bohnet, A. Safavi-Naini, M. L. Wall, J. J. Bollinger, and A. M. Rey, Nat. Phys. 13, 781 (2017).

[11] J. Li, R. Fan, H. Wang, B. Ye, B. Zeng, H. Zhai, X. Peng, and J. Du, Phys. Rev. X 7, 031011 (2017).
[12] J. S. Cotler, D. Ding, and G. R. Penington, Ann. Phys. (NY) 396, 318 (2018).

[13] C.-J. Lin and O. I. Motrunich, Phys. Rev. B 97, 144304 (2018).

[14] R. A. Jalabert, I. García-Mata, and D. A. Wisniacki, Phys. Rev. E 98, 062218 (2018).

[15] S. Pappalardi, A. Russomanno, B. Žunkovič, F. Iemini, A. Silva, and R. Fazio, Phy. Rev. B 98, 134303 (2018).

[16] K. X. Wei, C. Ramanathan, and P. Cappellaro, Phys. Rev. Lett. 120, 070501 (2018).

[17] A. Das, S. Chakrabarty, A. Dhar, A. Kundu, D. A. Huse, R. Moessner, S. S. Ray, and S. Bhattacharjee, Phys. Rev. Lett. 121, 024101 (2018).

[18] J. Rammensee and J. D. Urbina, and K. Richter, Phys. Rev. Lett. 121, 124101 (2018).

[19] I. García-Mata, M. Saraceno, R. A. Jalabert, A. J. Roncaglia, and D. A. Wisniacki, Phys. Rev. Lett. 121, 210601 (2018).

[20] C. W. von Keyserlingk, T. Rakovszky, F. Pollmann, and S. L. Sondhi, Phys. Rev. X 8, 021013 (2018).

[21] A. Nahum, S. Vijay, and J. Haah, Phys. Rev. X 8, 021014 (2018).

[22] V. Khemani, A. Vishwanath, and D. A. Huse, Phys. Rev. X 8, 031057 (2018).

[23] T. Rakovszky, F. Pollmann, and C. W. von Keyserlingk, Phys. Rev. X 8, 031058 (2018). 
[24] J. Chávez-Carlos, B. López-del-Carpio, M. A. BastarracheaMagnani, P. Stránský, S. Lerma-Hernández, and L. F. Santos, and J. G. Hirsch, Phys. Rev. Lett. 122, 024101 (2019).

[25] E. M. Fortes, I. García-Mata, R. A. Jalabert, and D. A. Wisniacki, Phys. Rev. E 100, 042201 (2019).

[26] M. Niknam, L. F. Santos, and D. G. Cory, Phys. Rev. Res. 2, 013200 (2020).

[27] F. Borgonovi, F. M. Izrailev, and L. F. Santos, Phys. Rev. E 99, 052143 (2019).

[28] R. Prakash and A. Lakshminarayan, Phys. Rev. B 101, 121108(R) (2020).

[29] P. D. Bergamasco, G. G. Carlo, and A. M. F. Rivas, Phys. Rev. Research 1, 033044 (2019).

[30] S. Pilatowsky-Cameo, J. Chávez-Carlos, M. A. BastarracheaMagnani, P. Stránský, S. Lerma-Hernández, L. F. Santos, and J. G. Hirsch, Phys. Rev. E 101, 010202(R) (2020)

[31] J. Ford, Phys. Today 36(4), 40 (1983).

[32] V. M. Alekseev and M. V. Jacobson, Phys. Rep. 75, 287 (1981).

[33] A. J. Lichtenberg and M. A. Lieberman, Regular and Chaotic Dynamics, 2nd ed. (Springer, New York, 1992).

[34] B. V. Chirikov, F. M. Izrailev, and D. L. Shepelyansky, Sov. Sci. Rev. C 2, 209 (1981).

[35] Y. Gu, Phys. Lett. A 149, 95 (1990).

[36] J. Ford, G. Mantica, and G. H. Ristow, Physica D 50, 493 (1991).

[37] Y. Gu and J. Wang, Phys. Lett. A 229, 208 (1997).

[38] A. K. Pattanayak and P. Brumer, Phys. Rev. E 56, 5174 (1997).

[39] J. Gong and P. Brumer, Phys. Rev. A 68, 062103 (2003).

[40] V. V. Sokolov, O. V. Zhirov, G. Benenti, and G. Casati, Phys. Rev. E 78, 046212 (2008).

[41] G. Benenti and G. Casati, Phys. Rev. E 79, 025201(R) (2009).
[42] V. Balachandran, G. Benenti, G. Casati, and J. Gong, Phys. Rev. E 82, 046216 (2010).

[43] T. Prosen, Phys. Rev. E 83, 031124 (2011).

[44] G. Benenti, G. G. Carlo, and T. Prosen, Phys. Rev. E 85, 051129 (2012).

[45] P. Qin, W.-G. Wang, G. Benenti, and G. Casati, Phys. Rev. E 89, 032120 (2014).

[46] G. Casati, J. Ford, and B. V. Chirikov, Phys. Lett. A 77, 91 (1980).

[47] More precisely, one should consider a generalized Lyapunov exponent, see [38,39]. Moreover, we do not consider here pathological cases like the baker's map for which orbits are exponentially unstable but Liouville phase-space evolution is exactly solvable [43].

[48] J. Wang, G. Benenti, G. Casati, and W. Wang, (unpublished).

[49] V. Bargmann, Commun. Pure Appl. Math. 14, 187 (1961).

[50] R. J. Glauber, Phys. Rev. 131, 2766 (1963).

[51] G. S. Agarwal and E. Wolf, Phys. Rev. D 2, 2161 (1970).

[52] See [48] for details on the numerics and, in particular, on a newly developed method enabling us to reliably compute in the classical case the number of harmonics up to quite long times, in spite of the complex phase-space structure.

[53] G. Berman and G. Zaslavsky, Physica A 91, 450 (1978).

[54] F. Haake, Quantum Signatures of Chaos, 2nd. ed. (SpringerVerlag, Berlin, 2000).

[55] M. Srednicki, J. Phys. A: Math. Gen. 32, 1163 (1999).

[56] T. Xu, T. Scaffidi, and X. Cao, Phys. Rev. Lett. 124, 140602 (2020).

[57] K. X. Wei, P. Peng, O. Shtanko, I. Marvian, S. Lloyd, C. Ramanathan, and P. Cappellaro, Phys. Rev Lett. 123, 090605 (2019).

[58] M. Gärttner, P. Hauke, and A. M. Rey, Phys. Rev. Lett. 120, 040402 (2018). 